\title{
Reduced-intensity conditioning allogeneic hematopoietic stem cell transplantation for chronic lymphocytic leukemia
}

\author{
Mohammad Faizan Zahid ${ }^{1,4} \cdot$ Natasha Ali $^{2,3}$
}

Received: 29 April 2015/ Accepted: 6 May 2015/Published online: 29 May 2015

(C) Springer Science+Business Media New York 2015

Chronic lymphocytic leukemia (CLL) is a complex hematologic malignancy following a highly variable clinical course [1]. Approximately one-third of CLL patients have an indolent disease course, surviving several years without needing therapy, whereas others suffer from aggressive disease and require treatment early in the disease process $[1,2]$. The combinations of new novel agents and targeted therapies with conventional chemotherapy have yielded better response rates CLL cases, but these therapies have proven inferior to allogeneic hematopoietic stem cell transplant (allo-HSCT) in terms of achieving long-term disease control and ultimately cure, especially in patients with relapsed/refractory disease [1-4].

The fact that allo-HSCT is capable of achieving longterm remissions in CLL patients, even in those with highrisk disease, supports the existence of the graft-versusleukemia (GvL) effect in CLL $[5,6]$, mediated by the alloreactive $\mathrm{T}$ lymphocytes in the donor graft [1]. This is further supported by the observation that the incidence of relapse decreases with time after $\mathrm{T}$ cell replete allo-HSCT [4], increased incidence of relapse after $\mathrm{T}$ cell-depleted allografting $[4,5]$, decreased relapse incidence in patients

Mohammad Faizan Zahid

faizanzahid91@ hotmail.com

Aga Khan University, Karachi, Pakistan

2 Department of Pathology \& Laboratory Medicine, Aga Khan University, Karachi, Pakistan

3 Department of Oncology, Aga Khan University, Karachi, Pakistan

4 House 54/1 - Sector E, Phase 1, Defence Housing Authority, Lahore, Pakistan who develop graft-versus-host disease (GvHD) than those who do not develop GvHD [3, 7] and the ability of donor lymphocyte infusions (DLI) to induce remarkable responses in patients with recurring/refractory disease posttransplant [1]. Notable advantages of allo-HSCT in comparison to autologous HSCT (auto-HSCT) are the infusion of a cancer-free stem cell graft from a healthy donor as well as the GvL effect, whereas auto-HSCT solely relies on the cytoreductive effect of myeloablative conditioning (MAC) regimens and is associated with high risks of subsequent relapse, persistent minimal residual disease (MRD) and secondary myelodysplasia, which outweigh the substantially low transplant-related mortality (TRM) associated with the procedure [2].

Present literature indicates that allo-HSCT is the only treatment modality that can offer a potential cure to CLL patients. However, TRM is significant, mostly from multiorgan damage, toxicity and high incidence of GvHD associated with MAC regimens [1]. TRM associated with MAC allo-HSCT has been reported to be as high as $46 \%$ $[1,2]$. A better understanding of the immunotherapeutic role of the GvL effect against CLL has allowed for the development of reduced-intensity conditioning (RIC) regimens in allo-HSCT, significantly reducing the toxicity and mortality that are associated with MAC regimens. Since CLL predominantly occurs in patients of advanced age, often those with multiple comorbidities, majority of them are not considered suitable candidates for MAC transplants [2]. RIC allows for a broader applicability of allo-HSCT in a larger number of these patients, significantly reducing TRM and yielding better outcomes with the procedure [1,4] by shifting some of the burden of leukemic cell kill from conditioning regimens to the GvL effect [2]. TRM associated with RIC allo-HSCT has been reported to be as low as $9.5-15 \%$ in literature $[3,4]$. 
Dreger et al. [6] reported the long-term follow-up results of the CLL3X trial conducted by the German CLL Study Group, which investigated the efficacy of RIC allo-HSCT in the treatment of CLL. The study involved 90 patients with poor-risk CLL, associated with poor prognostic factors such as early relapse after or refractoriness to purine analog-containing regimens, relapse after auto-HSCT and high-risk cytogenetic abnormalities such as unmutated IgVH gene, del(17p) and del(11q). The overall response rate from this report was $94 \%$ (73\% patients showing complete response). Quantitative analysis of MRD was performed in 52 patients, out of which $39(75 \%)$ remained progression-free and $27(52 \%)$ remained MRD-negative. The 4-year overall survival (OS) in the entire cohort was $65 \%$. Multivariate analysis showed that $\operatorname{del}(17 \mathrm{p})$ and purine analog refractory disease did not adversely affect response rates, progression-free survival (PFS) or OS, whereas $\mathrm{T}$ cell-depleted allografting was associated with shorter PFS and OS. Other studies have demonstrated that CLL exhibiting other high-risk factors (such as ZAP-70 overexpression, CD38 expression and partial remissions prior to transplant) is responsive to RIC allo-HSCT, indicating that RIC allo-HSCT can also overcome other poor prognostic factors $[1,8]$. In these studies, such poor-risk prognostic factors did not correlate with an increased risk of relapse. These findings indicated that RIC allo-HSCT is able to provide long-term disease control in CLL, despite poor-risk prognostic factors [6].

On the other hand, certain adverse features, such as bulky lymphadenopathy and purine analog refractoriness, predict an increased risk of relapse [1]. Data concerning the likelihood of relapse with purine analog refractory disease are controversial, as some phase 2 studies have indicated that allo-HSCT can offer long-term cure to even purine analog refractory disease, provided that patients show chemo-sensitivity to other agents [4]. In such patients, physicians should weigh the benefits and drawbacks of RIC and MAC regimens in terms of toxicity, organ damage and likelihood of relapse [1, 2]. Based on these observations, for patients to derive maximum benefit, allo-HSCT should be offered earlier in the course of the disease rather than exhausting all available therapeutic agents and before the onset of refractoriness $[4,8]$.

A recent retrospective study by Brown et al. [3] compared the efficacy of RIC allo-HSCT with MAC allo-HSCT for CLL. When comparing the entire patient cohort (timeline 1998-2009), there were no significant differences between PFS and OS of the two conditioning groups. With the introduction of the practice of performing RIC transplants early in CLL patients (as opposed to reserving transplant for only heavily pretreated patients with relapsed/refractory disease) and the development of better risk stratification systems for CLL patients, a subgroup analysis was carried out for patients treated 2004 onwards. This analysis showed that the two conditioning groups were more comparable for baseline characteristics in comparison with the cohort of the entire study period, allowing for a more objective comparison between the two conditioning groups. The results demonstrated a substantial OS advantage of RIC in comparison with MAC (5-year OS: 83 vs. $47 \%$ ), but similar PFS in both groups. RIC was also associated with significantly reduced TRM (9.5 vs. $46 \%$ for MAC) with comparable relapse rates (although a significant improvement was observed in relapse rate in RIC patients before and after 2004). Patients in the RIC group also benefited from reduced incidence of grade II-IV GvHD ( 30 vs. $50 \%$ for MAC). These findings demonstrated the superior efficacy of RIC allo-HSCT in the treatment of CLL, with better OS, comparable relapse rates and PFS and reduced toxicity, TRM and GvHD incidences when compared with MAC.

RIC allo-HSCT employs non-myeloablative doses of chemotherapeutic agents and consequently less cytoreductive capability in terms of tumor cell kill. Data concerning the risk of relapse after RIC transplant are controversial. While several studies suggest that reducing the ablative intensity of preparative regimens significantly reduces the short-term morbidity and overall mortality without affecting the GvL effect associated with alloHSCT, even in poor-risk CLL [5], others have demonstrated comparable [3] or increased risk of relapse posttransplant with RIC, offsetting its benefits [1, 7]. For example, one retrospective population-matched analysis [7] conducted by the EBMT comparing RIC with MAC demonstrated an increased incidence of disease relapse in the RIC group.

Applying flow cytometry and/or allele-specific oligonucleotide polymerase chain reaction to detect MRD before overt relapse occurs after allo-HSCT is a logical approach to maximize disease control and maintain remission in patients [1,9]. For those who are found to be MRD positive or show only partial response or relapse after allo-HSCT, the administration of DLI or withdrawal of immunosuppressants has shown promise by inducing molecular remissions (MRD-negative responses) in a majority of patients [6]. These findings support the role of preemptive MRD-guided immuno-modulation to improve patient outcomes by maximizing the GvL effect.

To the best of our knowledge, there are no prospective, randomized studies to determine the optimum conditioning regimen (RIC vs. MAC) for allo-HSCT in CLL. Most comparative studies published are limited to populationmatched analysis or retrospective, non-randomized comparisons $[1,4]$, with conflicting findings concerning the efficacy of RIC with MAC, especially in terms of relapse and PFS. It should be noted that studies involving RIC 
transplants are mostly prospective, multicenter studies involving large samples of patients and apply better risk stratification methods and residual disease monitoring in comparison to studies focusing on MAC allo-HSCT [5]. This indicates that the benefits and risks of RIC allo-HSCT in terms of TRM, relapse rate, PFS and OS needed to be objectively defined through a prospective, randomized trial design.

Since over two-thirds of CLL patients are individuals over 60 years of age, most of them having multiple comorbidities, it appears reasonable to offer RIC allo-HSCT to such patients for long-term disease control. Patients of younger age groups who are suitable candidates for MAC regimens can be offered either MAC or RIC for alloHSCT, provided that the benefits and risks of each treatment modality have been thoroughly considered. Patients should be referred for transplantation at the earliest possible time for the timely search for a suitable donor, to avoid excessive toxicity and organ damage associated with repeated chemo-immunotherapy and, most importantly, to maximize the benefit derived from the procedure before an eventual state of refractoriness after multiple rounds of treatment $[3,4]$.

Conflict of interest None.

\section{References}

1. Kharfan-Dabaja MA, Bazarbachi A. Hematopoietic stem cell allografting for chronic lymphocytic leukemia: a focus on reduced- intensity conditioning regimens. Cancer Control. 2012;19(1): 68-75.

2. Khouri IF. Reduced-intensity regimens in allogeneic stem-cell transplantation for non-hodgkin lymphoma and chronic lymphocytic leukemia. Hematol Am Soc Hematol Educ Program. 2006;1:390-7.

3. Brown JR, Kim HT, Armand P, et al. Long-term follow-up of reduced-intensity allogeneic stem cell transplantation for chronic lymphocytic leukemia: prognostic model to predict outcome. Leukemia. 2013;27(2):362-9.

4. Mewawalla P, Nathan S. Role of allogeneic transplantation in patients with chronic lymphocytic leukemia in the era of novel therapies: a review. Ther Adv Hematol. 2014;5(5):139-52.

5. Dreger P, Corradini P, Kimby E, et al. Indications for allogeneic stem cell transplantation in chronic lymphocytic leukemia: the EBMT transplant consensus. Leukemia. 2007;21(1):12-7.

6. Dreger P, Döhner H, Ritgen M, et al. Allogeneic stem cell transplantation provides durable disease control in poor-risk chronic lymphocytic leukemia: long-term clinical and MRD results of the German CLL Study Group CLL3X trial. Blood. 2010;116(14):2438-47.

7. Dreger P, Brand R, Milligan D, et al. Reduced-intensity conditioning lowers treatment-related mortality of allogeneic stem cell transplantation for chronic lymphocytic leukemia: a populationmatched analysis. Leukemia. 2005;19(6):1029-33.

8. Khouri IF, Saliba RM, Admirand J, et al. Graft-versus-leukaemia effect after non-myeloablative haematopoietic transplantation can overcome the unfavourable expression of ZAP-70 in refractory chronic lymphocytic leukaemia. Br J Haematol. 2007;137(4): 355-63.

9. Moreno C, Villamor N, Colomer D, et al. Clinical significance of minimal residual disease, as assessed by different techniques, after stem cell transplantation for chronic lymphocytic leukemia. Blood. 2006;107(11):4563-9. 